\title{
Annual Conference of Blood Purification Center Management Branch of Chinese Hospital Association
}

August 3-16, 2017, Beijing, China

\section{Selected Abstracts}

Guest Editor

Tao Wei, Beijing 
1

\section{Hepatitis C Virus Outbreak in a Hemodialysis Unit of a Primary Hospital: Infection Investigation and Treatment Follow-Up}

Hua Liü1, Jinhong Xue ${ }^{\# 1}$, Fahui Zhao ${ }^{2}$, Julin Gao', Yingli He1, Nan Guo², Hongli Jiang*1

${ }^{1}$ Department of Blood Purification, First Affiliated Hospital, Xi'an Jiaotong University, Xi'an, Shaanxi, China; ${ }^{2}$ Department of Infectious Diseases, First Affiliated Hospital, Xian Jiaotong University, Xi'an, Shaanxi, China; 'Zhen'an People's Hospotal, Shangluo, Shaanxi, China

*Corresponding authors: Hongli Jiang,

Email: j92106@sina.com

\#Both authors equally contributed as first authors

Objective: In January and Match 2016, 45 patients at a hemodialysis unit of a primary hospital were given a diagnosis of new hepatitis $\mathrm{C}$ virus (HCV) infection. The outbreak of $\mathrm{HCV}$ infection was investigated to identify risk factors for transmission and the treatment was followed up.
Methods: We monitored the HCV-RNA situation and HCV genotyping of 45 patients with positive anti-HCV antibody. The epidemiological investigation, infection possible risk factors were analyzed, and treatment response and tolerance of different patients were followd-up.

Results: From the analysis of various aspects of hemodialysis including factors of HCV transmission: 1) Regulations on the use of disposable articles are not strictly enforced; 2) The infection consciousness of medical staff is dim, and the conception of asepsis is not strong; 3) the hospital system is not perfect; 4) Infection index monitoring is defective. In the 45 infected patients, the genotype $2 \mathrm{a}$ were 32 cases $(71.11 \%), 1$ b were 8 cases $(17.78 \%)$, and 2 a mixed 1 b were 4 cases $(8.89 \%), 1(2.22 \%)$ patients did not measure genotype has started treatment, overall genotype proportion is different from the common HCV mainly in $1 \mathrm{~b}$ type in China. 22 patient (48.9\%) used the protocol of interferon and ribavirin (PR protocol) and 2 for interferon alone at the beginning, but 21 patients of those were changed to the direct-acting antiviral agents (DAAs) protocol because of the side effect by PR protocol after we acquired the Sofosbuvir and Daclatasvir from Laos. 18 cases used the DAAs protocol at the beginning. All the 41 patients used the DAAs protocol (Sofosbuvir $200 \mathrm{mg}$ and Daclatasvir $60 \mathrm{mg}$ for hemodialysis patients and Sofosbuvir $400 \mathrm{mg}$ and Daclatasvir $60 \mathrm{mg}$ for undialysis

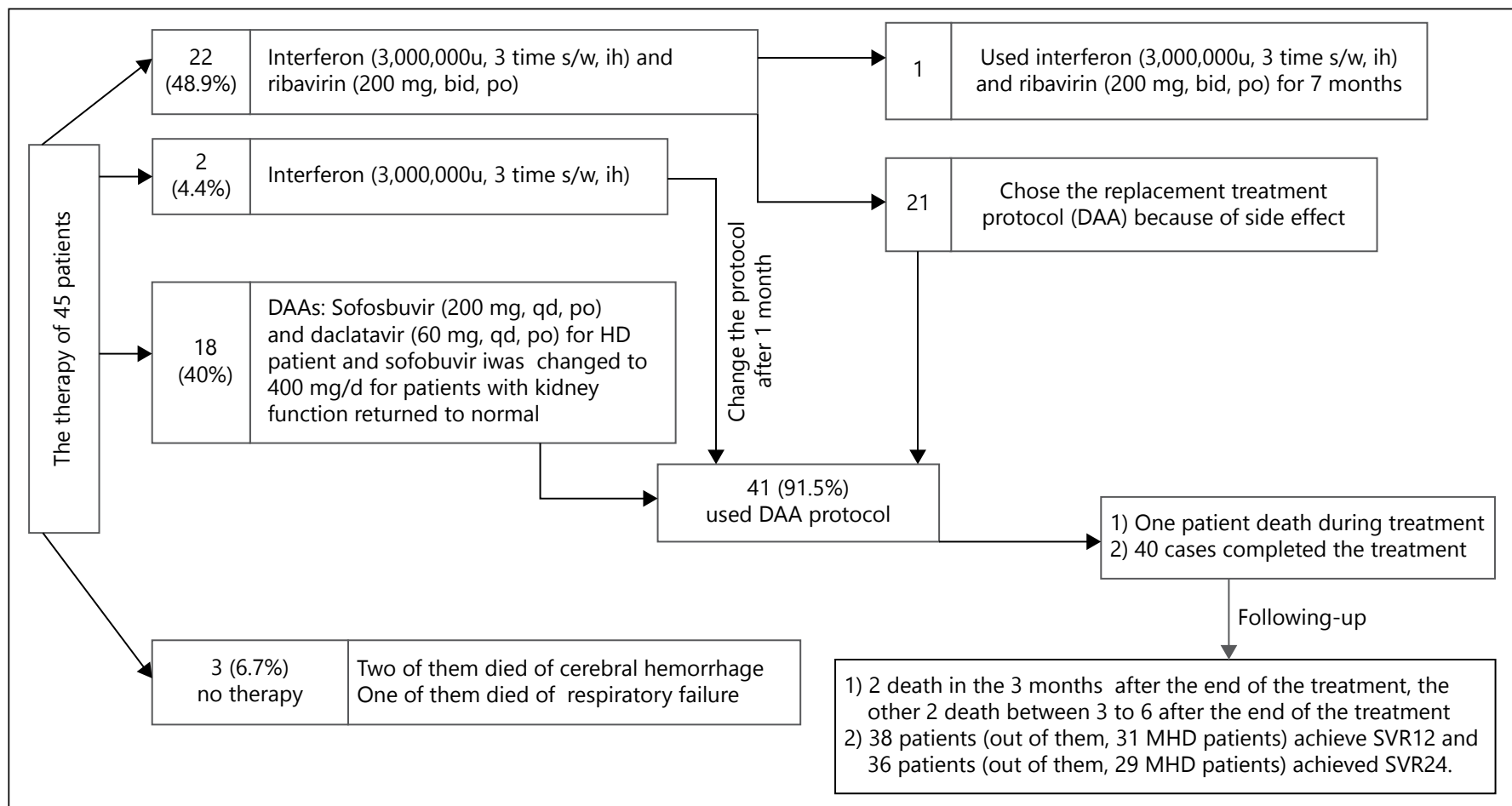

Fig. 1. The therapy of 45 patients. 
patients), not only first using but also as the replacement, there were no patient exiting because of the intolerance. 5 patients died during or after the end of the treatment (unrelated to the treatment) and 38 patients used DAAs had obtained sustained virological response (SVR) after Stop drug discontinuation 12 weeks (SVR12, 38/38) and 36 cases achieved SVR24 (36/36). The therapy of 45 patients were shown in Figure 1.

Conclusions: Strengthen the control measures, and strictly implement the infection control system is an important measure to prevent hemodialysis patients from HCV infection. DAAs scheme (Sofosvuvir + Daclatasvir) for HD patient with HCV-infection has better tolerance, which is maybe a good choice for patients with intolerance of PR protocol, and have better antiviral effect, but also need a lot of guidance for clinical use in clinical data support.

Key Words: Hepatitis C virus, Hemodialysis, Outbreak, Treatment.

\section{2 \\ High-Phosphotus-Induced VSMCs Calcification Mediated by BMP-2 Signaling Pathway}

Huiran Zhang, Jinsheng Xu*, Liping Guo, Yaling Bai, Shenglei Zhang, Junxia Zhang, Liwen Cui

Department of Nephrology, The Forth Hospital of Hebei Medical University, Shijiazhuang, China

Corresponding author: Jinsheng Xu,

Email:xjs5766@126.com

Objective: To explore the effect of different $\mathrm{PH}$ on calcification of rat vascular smooth muscle cells (VSMCs) through the bone morphogenetic protein2 (BMP-2) signaling pathway.

Methods: Healthy male SD rats aged 8-10 weeks were selected in the study. VSMCs from rat thoracic aorta were cultured in vitro, and then identified by immunocytochemistry. The VSMCs were randomly divided into 5 groups by random sampling method: normal group (pH7.4), pH7.4+high phosphorus group, $\mathrm{pH7.1+high} \mathrm{phos-}$ phorus group, $\mathrm{pH} 7.7+$ high phosphorus group, and inhibitor group. Calcium deposition and ALP activity were measured by Alizarin red staining, quantification of calcium and enzyme linked immunosorbent assay. The expression of BMP-2, Smad1, Runx2 mRNA was detected by RT-PCR and Western blot.

Results: Compared with the control group, the $\mathrm{pH} 7.4+$ high phosphorus group calcification staining, calcium content increased $(P<$ $0.05)$. While compared with the $\mathrm{pH} 7.4+$ high phosphorus group, the $\mathrm{pH} 7.1+$ high phosphorus group calcification staining, calcium content and alkaline phosphatase (ALP) activity and the expression of BMP2, Smad1, Runx2 average decreased $(\mathrm{P}<0.05)$, and the $\mathrm{pH} 7.7+$ high phosphorus group calcification staining, calcium content and alkaline phosphatase (ALP) activity and the expression of BMP-2, Smad1, Runx2 average increased $(\mathrm{P}<0.05)$, Compared with the $\mathrm{pH} 7.4+$ high phosphorus group, the expression of Smad1 and Runx2 in inhibitor group also decreased $(\mathrm{P}<0.05)$.

Conclusion: Extracellular acidic environment can inhibit highphosphotus-induced VSMCs calcification, whereas extracelluar alkaline environment induce high-phosphotus-induced VSMCs cal- cification. The mechanism is presumably that VSMCs calcification is induced by influencing BMP-2 pathway, which may mediate the transdifferentiation of vascular smooth muscle cells.

Key Words: High-phosphotus, Vascular smooth muscle cells (VSMCs), Calcification, Bone morphogenetic protein 2.

\section{3 \\ Low Time-Average iPTH is an Independent Risk Factor for Adverse Outcomes in Peritoneal Dialysis Patients}

\author{
Bei Wu, Huiping Zhao, Mei Wang \\ Department of Nephrology, Peking University People's \\ Hospital, Beijing, China
}

Objective: KDIGO guideline recommended iPTH target range between 2-9 times normal value. Whether low iPTH is an independent risk factor for all-cause death is not clear.

Methods: Clinical date were analyzed for all adult patients (age $>18$ years) with chronic renal failure who had commenced peritoneal dialysis between January, 2006 and April, 2014 in our Hospital.

Results: There were 235 patients enrolled, 118 in low iPTH (iPTH $<150 \mathrm{pg} / \mathrm{ml}$ ) group, 117 in non-low iPTH (iPTH $\geq 150 \mathrm{pg} / \mathrm{ml}$ ) group. Mean follow-up time was 41.5 months. Compared to the nonlow iPTH group, the low iPTH group was older $(63 \pm 14$ years vs. $58 \pm 14$ years, $\mathrm{P}=0.001)$, more male $(54 \%$ vs. $42 \%, \mathrm{P}=0.039)$, more diabetes $(57 \%$ vs. $42 \%, \mathrm{P}=0.001)$, higher Charlson complication index $(3.77 \pm 1.22$ vs. $3.36 \pm 1.24, \mathrm{P}=0.011)$, higher eGFR $(8.04 \pm$ $3.87 \mathrm{ml} / \mathrm{min} / 1.73 \mathrm{~m}^{2}$ vs. $6.86 \pm 3.73 \mathrm{ml} / \mathrm{min} / 1.73 \mathrm{~m}^{2}, \mathrm{P}=0.018$ ) when they started dialysis. During follow-up period, the low iPTH group had lower time-average diastolic pressure $(75 \pm 10 \mathrm{~mm} \mathrm{Hg}$ vs. $78 \pm$ $10 \mathrm{~mm} \mathrm{Hg}, \mathrm{P}=0.007)$, lower time-average daily total output volume (982 $\mathrm{ml}$ vs. $1094 \mathrm{ml}, \mathrm{P}=0.044$ ) and lower time-average serum phosphorus ( $1.41 \pm 0.27 \mathrm{mmol} / \mathrm{L}$ vs. $1.50 \pm 0.27 \mathrm{mmol} / \mathrm{L}, \mathrm{P}=0.009)$, compared to the non-low iPTH group. The 1-, 2-, 3-, 4- and 5-year survival rates of low iPTH PD patients were $92.4 \%, 78.8 \%, 73.7 \%$, $64.4 \%$, and $61.0 \%$, respectively. The 1-, 2-, 3-, 4- and 5-year survival rates of non-low iPTH PD patients were 97.4\%, 90.6\%, 85.5\%, $82.1 \%$, and $77.8 \%$, respectively. Low iPTH group had a worse survival compared to the non-low iPTH group. And, when adjusted for age, gender, diabetes, CCI, eGFR, diastolic pressure, daily total output volume, and serum phosphorus, there was still significant difference in survival between low iPTH group and non low iPTH group.

Conclusion: Low time-average iPTH is an independent risk factor for adverse outcomes in peritoneal dialysis patients.

Key Words: Peritoneal dialysis, iPTH, Survival rate. 


\section{4}

\section{The Effects of the Intra-Abdominal Place of the Peritoneal Dialysis Tube in B Ultrasound Detection on Complications}

\author{
Min Yang, Liu Yang \\ Department of Nephrology, Union Hospital, Tongji Medical \\ College, Huazhong University of Science and Technology, \\ Wuhan, China \\ Corresponding author: Min Yang \\ E-mail: yangminym83@126.com
}

Objective: Peritoneal dialysis has been recognized as one of effective alternative therapies for chronic renal failure (CRF). Currently, the common method is to place the end of tube into the true pelvis (pouch of Douglas) i.e. vesicorectal pouch or uterorectal pouch. As for the depth of catheterization and the relations between it and complications were without definitive conclusion. There are several methods of catheterization and body surface location both home and abroad without definitive conclusion. We plan to do preliminary study to investigate the influence of distance between the end of the intra-abdominal part of the peritoneal dialysis tube and the bottom of pouch of Douglas on the complications, under the guidance of B ultrasonography.

Material and Method: We selected 112 patients who were hospitalized in our department from June, 2014 to June 2016 as our research subjects. Among them, there are 64 males aging from 19-74. All the 112 subjects have clear indications for kidney disease alternative therapy without contraindications to peritoneal dialysis. 112 patients were divided by gender. The catheterization location for all subjects was a surgical incision made $2-3 \mathrm{~cm}$ the right of the median line below the umbilicus. In one week after catheterization, detection was made under the guidance of B ultrasonography. Before detection, the patients should be asked to perform evacuation. Then $1 \mathrm{~L}$ of $1.5 \%$ peritoneal dialysis solution was injected into the abdominal cavity according to the operation principles of peritoneal dialysis. And guidance should be given to the patients to get rid of unnecessary stress, which will facilitate the detection of the distance between the end of the intra-abdominal part of the peritoneal dialysis tube and the bottom of pouch of Douglas. We divided the patients into three groups according to the distance-Group $1(0-3 \mathrm{~cm})$, Group $2(3-5 \mathrm{~cm})$, and Group $3(>5 \mathrm{~cm})$. Preliminary judgment was made about the appropriate catheterization method after the data was collected about the numbers of patients who had complications after catheterization in the three groups. The data collected was processed by the statistical software SPSS 13.0.

Results: The difference was significant $(\mathrm{P}<0.05)$ in the prevalence of catheterization complications among the three groups regardless of gender. The prevalence of Group $2(3-5 \mathrm{~cm})$ is the lowest, better than other two groups. A follow-up of the 112 patients was made which lasted one to two years. The study has found that when the distance (between the end of the intra-abdominal part of the peritoneal dialysis tube and the bottom of pouch of Douglas) was $0-3 \mathrm{~cm}$, patients were more likely to have abdominal pain at a serious level, and the pain in part of the patients would not be alleviated after 2 years of dialysis; And when the distance $>5 \mathrm{~cm}$, patients were more likely to have complications of tube floating and tube plugging, most of which need extubation and resetting a tube or changing to hemodialysis; Only when the distance is $3-5 \mathrm{~cm}$, the prevalence of the four complications mentioned above would decrease significantly with statistical significance.

Conclusion: Therefore placing the peritoneal dialysis catheter in the ideal position to decrease the prevalence of complications is one of the pressing issues we need to address in our clinical practices of peritoneal dialysis catheterization. To enable every peritoneal dialysis patient enjoy the benefits of the high-quality peritoneal dialysis, we need to consistently improve the technology of peritoneal dialysis catheterization, thus promoting the harmony in families as well as the whole society.

Key Words: B ultrasound, Peritoneal dialysis tube, Intraabdominal place, Complications.

\section{5 \\ Effects of Two Disinfectants on Preventing Central Line Associated Blood Stream Infection}

\author{
Fan Zhang, Youwen Xiao, Oin Wang \\ Blood Purification Center of Nephrology Department of \\ Leshan People's Hospital, Sichuan Province, China \\ Corresponding author: Fan Zhang \\ Email:387394@qq.com
}

Objective: To probe into the effects of two disinfectants on preventing central line associated blood stream infection (CLABSI).

Methods: 168 patients receiving blood purification through central venous indwelling catheter in our hospital from November 2015 to January 2017 were selected in this controlled clinical research, and they were randomly divided into the control group and observation group. $5000 \mathrm{mg} / \mathrm{L}$ iodophor disinfectant and chlorhexidine gluconate were adopted to disinfect sites of catheterization and catheter nursing, respectively; differences in skin colony count and CLABSI occurrence between two groups were compared before central venous catheterization, immediately and $24 \mathrm{~h}$ after central venous catheterization.

Result: No statistical significance in the skin colonies after inserting central venous catheter was observed between observation group and control group before disinfection, while the skin colonies in observation group $24 \mathrm{~h}$ after disinfection was lower than that of control group. According to CLABSI diagnostic criteria, the morbidity of CLABSI in observation group was lower than the controls, and the difference was statistically significant $(\mathrm{p}<0.05)$.

Conclusion: The application of chlorhexidine digluconate lowered the morbidity of bloodstream infection induced by hemodialysis catheter, and the antibacterial duration was longer than povidone iodine, which was worthy of promoting in clinical practice.

Key Words: Chlorhexidine gluconate, Central line associated blood stream infection (CLABSI), Hemodialysis catheter. 


\section{6}

\section{Study on Training System of Blood Purification Specialist Nurses in Shanxi Province}

\author{
Julin Gao, Xiaomin Liu, Qiongjie Li, Xiaohong Xue, Xia Xin \\ Blood Purification Department, The First Affiliated Hospital \\ of Xi'an Jiaotong University, Jiaotong, China
}

Objective: To construct the training system of blood purification specialist nurses in Shanxi Province, and to train qualified blood purification specialist nurses for primary hospitals.

Methods: In order to develop the training system of blood purification specialist nurses in Shanxi Province, we established a blood purification specialist nurse training institutions, and through literature researches, questionnaire surveys, experience summaries and so on to develop the training system of blood purification specialist nurses in Shanxi. We carried out an 8-week of theoretical and operational training for nurses of the whole province, and after passing the final examination at the end of the training, then the learners returned to guide the work of their own hospital. We summarized at the end of each trainee training, and constantly improved the training and management system.

Result: From 2014 to 2016 a total of 302 nurses were trained in blood purification. The average score of the theory test was increased from 64.47 points before training to the score of 92.3 after training $(\mathrm{P}<0.001)$; The average score of self evaluation of teaching and research ability was increased from 40.38 points before training to the score of 82.35 after training $(\mathrm{P}<0.001)$; The evaluation of the effectiveness of the training institution to the specialist nurses was increased from 88.2 points before training to the score of 90.2 after training $(\mathrm{P}<0.001)$. Compared with before training, there was statistical significance after training.

Conclusion: Through the training of blood purification specialist nurses, the theoretical knowledge, teaching and scientific research ability and comprehensive quality of hemodialysis nurses were improved significantly, and this provides a strong guarantee for the establishment of a specialist nursing team of Shanxi province.

Key Words: Blood purification, Specialist nurse, Training system.

\section{7 \\ The Effect of Surgical Intervention of Dorsal Hand Branch of Cephalic Vein on Radial Artery Cephalic Vein Fistula}

\author{
Xiangfei Yao ${ }^{1}$, Qiaoning Wei ${ }^{2}$ \\ ${ }^{1}$ Kidney Medicine of Xi'an Gaoxin Hospital, Gaoxin, China; \\ ${ }^{2}$ Blood Purification Department of the First Affiliated \\ Hospital of xi'an Jiaotong University, Jiaontong, China
}

Objective: Perform ligation of cephalic vein on the dorsum of the hand to observe the influence on inner diameter of the cephalic vein and prevent stenosis or occlusion of arteriovenous fistula (AVF) in cephalic vein or even swollen hand syndrome due to reversed blood flow in cephalic vein on the dorsum of the hand.
Methods: 150 cases of radial artery-cephalic vein fistula with reversed flow in cephalic vein on the dorsum of the hand were divided into three groups, with those of intraoperative surgical intervention in cephalic vein on the dorsum of the hand as Group A, postoperative surgical intervention in arterialized cephalic vein on the dorsum of the hand as Group B and the unoperated ones as Group C. The patients had received observation of inner diameters of their main cephalic veins for 2 years to record the occurrence of stenosis, occlusion or swollen hand syndrome of the main cephalic veins.

Results: 1 . Through observation, the inner diameters of cephalic veins of patients in Group $\mathrm{C}$ were reduced over time according to the AVF preoperative ultrasonic Doppler testing results and postoperative measurements in 1-2 years, which indicated statistical significance $(\mathrm{P}<0.05)$. There were no differences in preoperative condition among the three groups $(\mathrm{P}>0.05)$. During the first year after operation, statistical differences between Group A and Group B and C were found $(\mathrm{P}<0.05)$ while Group $\mathrm{B}$ and Group $\mathrm{C}$ also showed differences $(\mathrm{P}<0.05)$; In 2 years after operation, there were differences between Group A and Group B and C $(\mathrm{P}<0.05)$ and meanwhile, differences were seen between Group B and Group C $(\mathrm{P}<0.05)$. Each group respectively showed significant differences in the condition before operation and in one and two years after operation $(\mathrm{P}<0.01)$, which was associated with the blood flow from an artery to a vein through radial artery-cephalic vein fistula. 2 . The three groups had significant differences in the occurrence of stenosis, occlusion and swollen hand syndrome, which indicated statistical significance $(\mathrm{P}<0.01)$. Among the three groups, patients of Group C, followed by Group B, were most likely to have these complications whereas Group A had the lowest incidence.

Discussions: Patients with radial artery-cephalic vein fistulas on the dorsa of their hands should receive ligation as early as possible to block reversed blood flow and prevent stenosis and occlusion of cephalic veins and swollen hand syndrome.

Key Words: Surgical intervention, The head of the surgical vein, The radial artery - the internal fistula of the head (AVF).

\section{8}

\section{The Associated Factors with Bone-Specific Alkaline Phosphatases in Maintenance Hemodialysis Patients}

\author{
Zuanhong Jiang, Zljin Chen, Zhiyu Wang, Xiaobo Ma, \\ Chunli Zhang, Ying Qian, Zhaohui Wang, Haijin Yu, \\ Xiaonong Chen \\ Department of Nephrology, Ruijin Hospital, Shanghai \\ Jiaotong University School of Medicine, Shanghai, China \\ Corresponding author: Xiaonong Chen \\ Email: xiaonong@medmail.com.cn
}

Objective: Serum bone-specific alkaline phosphatases (BAP) is a new biological marker of chronic kidney disease-mineral and bone disorder (CKD-BMD). The aim of this study was to analysis serum BAP level and associated factors.

Methods: Uremia patients received hemodialysis treatment in Ruijin Hospital were recruited in July 1, 2012. Inclusion criteria for this study were as follows: 18 80 years old, dialysis vintage of at least 3 months. All clinical data, biochemical data and medication 
were collected at baseline. Bood samples of MHD patients and 35 healthy volunteers were collected to detect serum BAP level by the MicroVue BAP immunoassay (Quidel, San Diego, CA).

Results: Two hundreds and nineteen MHD patients were involved in this study with a median age of 58.00 (interquartile range, 48.00-67.00) years old, hemodialysis duration of 41.00 (interquartile range, $21.00-76.00)$ months, and $10.96 \%$ with diabetes. Median levels of BAP were 19.67 (14.83-26.98) U/L. No significant differences were found in serum BAP levels of MHD female patients versus female controls, but the BAP levels of MHD male patients were significantly lower than male controls. In MHD patients, serum BAP levels were significantly higher in female than in male. There were positive correlations between ALP, parathyroid hormone (PTH), BAP/ALP, hemodialysis duration, single-pool Kt/V (spKt/V), total iron binding capacity (TIBC) and BAP. Blood bicarbonate level and post-dialysis systolic blood pressure were negatived associated with BAP. Multivariate logistic regression showed that female, using $\beta$-blocker, and higher PTH level were independently correlated with BAP.

Conclusion: BAP is a sensitive marker of bone metabolism in MHD patients. Those who are female, with treatment of $\beta$-blocker and higher PTH level may have a higher serum BAP levels.

Key Words: Chronic kidney disease, Maintenance hemodialysis, Chronic kidney disease-mineral and bone disorder, Bone alkaline phosphatase, Associated factors.

\section{9 \\ Multifaceted Assessment of Nutritional Status in Maintenance Hemodialysis Patients}

Mei Han, Si Sun, Jiameng Li, Oiang Liu, Tingting Yang, Baihai Su

Division of Nephrology, Department of Medicine, West

China Hospital of Sichuan University, Chengdu, China

Corresponding author: Baihai Su

Email: imsbh@163.com

Objective: To investigate the efficiency of bioelectrical impedance analysis (BIA), Laboratory index, body mass index (BMI), nutrition scale assessment methods in evaluating the nutritional status of maintenance hemodialysis (MHD) patients.

Methods: 536 maintenance hemodialysis patients without the severe hepatic insufficiency, acute severe infection, malignant tumors and severe edema in West China Hospital Dialysis Center were included in the study. The nutritional status of the patients was evaluated by The BIA (body cell mass (BCM), body fat percentage (PBF), fat free mass (FMM), Laboratory index (albumin and triglyceride) and nutrition scale assessment (the malnutrition inflammation score (MIS) and the modified quantitative subjective global assessment (MQSGA)) were used to evaluate the patient's nutritional status.

Results: The average levels of indexes in patients were body cell mass $(\mathrm{BCM})$ for $(28.68+6.01) \mathrm{kg}$, body fat percentage $(\mathrm{PBF})$ for $(24.31+10.01) \%$, fat free mass $(\mathrm{FMM})$ for $(44.00 \pm 8.90) \mathrm{kg}$, MIS for $(4.76+2.07)$, MQSGA for $(10.98+1.61)$, BMI for $(21.93+3.18)$, ALB for $(41.45+3.83) \mathrm{g} / \mathrm{L}$, TG for $(1.42+0.97) \mathrm{mmol} / \mathrm{L}$, which $45.52 \%$ BCM, 18.10\% BMI, 35.26\% ALB (patients with ALB below
$35 \mathrm{~g} / \mathrm{L}$ accounted for $7.09 \%), 0.93 \%$ TG were lower than the normal levels. The malnutrition (including malnutrition risk) of patients assessed by $\mathrm{BCM}$ was $45.52 \%$, significantly higher than $\mathrm{BMI}(\mathrm{P}<$ $0.001)$, ALB $(\mathrm{P}<0.001)$ and TG $(\mathrm{P}<0.001)$. BCM may be an early indicator of malnutrition in MHD patients. The result of correlation analysis showed that the BCM was positively correlated with ALB, $\mathrm{Hb}$, BMI, Scr and FFM, and negatively correlated with gender, age, PBF, MIS, while age, TG, MQSGA had no correlation. We controlled the gender, age and diabetes factors, the relationship between BCM and ALB, Hb was disappeared, and has a positive correlation with BMI, Scr, FFM, negative correlation with MIS.

Conclusion: BCM, ALB, Scr, BMI, FFM and MIS can effectively evaluate the nutritional status of maintenance hemodialysis patients; and the BCM may be the early indicators of malnutrition, which also has the advantages of simple operation, noninvasive, inexpensive, long-term repeated measurement and has the wide clinical application prospect.

Key Words: Hemodialysis, Nutrition, Bioelectrical impedance analysis, Body composition analysis.

\section{0 \\ Comparison of the Early Cannulation Graft (Gore Acuseal) and Standard Graft (Gore Intering) for Prosthetic Vascular Access for Haemodialysis \\ Yong $X u^{1}$, Bing Tang ${ }^{2}$, Yuanming $L i^{1}$, Xinxin $L i u^{2}$, Kun Wu ${ }^{1}$ \\ ${ }^{1}$ Hemodialysis Center, The Third Affiliated Hospital of \\ Xiang Ya School of Medicine, Central South University, \\ Changsha, China; ${ }^{2}$ Renal Division, Department of \\ Medicine, Jewel Hospital, Changsha, China \\ Corresponding author: Kun Wu \\ E-mail:wukun@jewelmedical.com}

Objective: Gore Acuseal is a new early cannulation prosthetic access, it can be cannulated for dialysis within 3 days. The characteristic of this prothetic access is an attractive alternative to CVC in those requiring urgent dialysis patients. The purpose is to compare the safety and efficacy of early cannulation graft (Gore Acuseal) to standard graft (Gore Intering) for prosthetic vascular access for Haemodialysis.

Methods: This is a prospective observational study of all AVGs placed since December 2016 to June 2017 in our hospital. Outcomes including time to first cannulation, patency rate, rates of seroma, access thrombosis, steal syndrome, pseudo-aneurysm and infection in early cannulation graft (Gore Acuseal) comparison to standard Grafts (Gore Intering).

Results: Sixteen Gore Acuseal grafts and nineteen Gore Intering grafts were implanted in the study period. The Gore Acuseal graft configuration was superficial femora-saphenous $(n=15)$, upper arm axillo-axillary $(\mathrm{n}=1)$; The Gore Intering graft configuration was superficial femora-saphenous $(\mathrm{n}=15)$, upper arm brachial-axillary $(\mathrm{n}=1)$, brachial-cephalic or basilic $(\mathrm{n}=3)$. No patient was lost during a mean follow-up time of $3.6 \pm 1.6$ months (rang, 1.7-6.0 months). Primary functional patency (Gore Acuseal $100 \%$ vs Gore Intering $73 \%)$, Secondary patency rate $(93.8 \%$ vs $89.5 \%)$. Mean time to first cannulation was $5.4 \pm 4.0$ days (rang, $1-16$ days) vs $28.0 \pm 4.1$ days 
Table 1. Statistics of equipment failure, maintenance cost and vacancy time in 2015-2016 and 2016-2017

\begin{tabular}{lrrr}
\hline & 2015 & 2016 & Drop than \\
\hline The number of maintenance & 137 & 95 & $30.65 \%$ \\
Predictive maintenance times & 104 & 67 & $35.57 \%$ \\
Unpredictabable maintenance times & 33 & 28 & $15.15 \%$ \\
Maintenance cost (¥) & 43,456 & 13,277 & $69.44 \%$ \\
Equipment vacancy time (minutes) & 24,633 & 11,602 & $52.90 \%$ \\
\hline
\end{tabular}

* The time of vacancy was in accordance with the time for engineers to get to the site during equipment maintenance or delivery time of parts. The main city was calculated according to 4 hours; The field was calculated in 24 hours.

(rang, 21-35 days), the differences were statistical significance $(\mathrm{p}<$ 0.05 ). Seroma ( 0 vs $64.2 \%, p<0.05)$, AV access infection ( 0 vs $21 \%$ ). Steal syndrome occurred in one patient with Gore Acuseal graft. Primary blood flow rate has no difference in both grafts.

Conclusion: Comparing with Gore Interning, Gore Accused Graft implantation was safe and effective, early cannulation with higher patency rate, less seroma, thrombosis, and infection. It is a viable option for patients who require urgent hemodialysis instead of temporary or tunneled catheters.

Key Words: Gore accused graft, Gore interning graft, Haemodialysis.

\section{1}

\section{The Application and Significance of Predictive Maintenance in Dialysis Equipment Management}

\author{
Long Huang, Yunyan Wang, Lei Feng, Jing Mao \\ The Third Military Medical College Affiliated to Daping \\ Hospital in the Department of Renal Internal Medicine \\ Blood Purification Center Chongqing, China \\ Corresponding author: Yunyan Wang \\ E-mail: 78905052@qq.com
}

Objective: To explore the application and importance of predictive maintenance in dialysis devices

Methods: The study subjects were 57 dialysis machines in my center, and the dialysis machine was used for 3 to 10 years. The brand models included B.braun Dalog+ and Dalong+-online, AshiKASEI MDS-101 and GAMBRO Ak-95s.

Predictive Maintenance Methods: through the device of individualized maintenance records, repair and maintenance of its nearly three years for statistical data, summed up the failure rate of each device and parts replacement cycle, work out a plan for each of the predictive maintenance. By retrospective study, the 57 dialysis equipment in our center was used with the method of predictive maintenance for one year from 2016 to 2017, which was not used with this method for one year from 2015 to 2016. Recorded the numbers of equipment failure, predictable failure, unforeseen fault, maintenance costs and save equipment idle time during the two period
(2015-2016 and 2016-2017). Compared the relevant data between the two period.

Results: After adopting predictive maintenance, equipment failure frequency, the number of predictable failure and the maintenance cost dropped significantly. Equipment vacancy time also dropped obviously (see table 1). There was no significant change in the number of unforeseen failures.

Conclusion: 1 . The replacement cycle of equipment parts has a great effect on reducing the failure rate of equipment.

2. The summary and implementation of maintenance frequency can effectively improve equipment failure.

3 Predictive maintenance plays an important role in reducing equipment failure rate and prolong the service life of the equipment. It can greatly reduce the probability of failure in equipment operation, improve the safety of patients and patients' satisfaction, save equipment empty time and improve equipment efficiency.

Key Words: Predictive maintenance, Dialysis equipment management.

12

\section{CSE/H2S System Alleviates Uremic Accelerated Atherosclerosis Development by Regulating TGF- $\boldsymbol{\beta} /$ Smad Pathway}

Han Li, Su-juan Feng, Xiang-xue Lu, Rui-fang Xiong, Shi-xiang Wang*

Department of Blood Purification, Beijing Chao-Yang

Hospital, Capital Medical University; Nephrology Faculty,

Capital Medical University, Beijing, China

*Corresponding author: Shi-xiang Wang

E-Mail: sxwang@ccmu.edu.cn

This work was supported by National Natural Science Foundation of China (81670673), National Natural Science Foundation of China (81200543) and Beijing Natural Science Foundation (7142057).

Objective: Cystathionine- $\gamma$-lyase (CSE) produces hydrogen sulfide $\left(\mathrm{H}_{2} \mathrm{~S}\right)$ in the cardiovascular system, which can inhibit the atherosclerosis development and progression, including gatherogenic modification of LDL, monocytes adhesion to the endothelial cells, macrophage-derived foam cell formation and inflammation, smooth 
muscle cell proliferation, neointimal hyperplasia, vascular calcification, and thrombogenesis. Our previous study has demonstrated that $\mathrm{CSE} / \mathrm{H}_{2} \mathrm{~S}$ play an important role in uremic accelerated atherosclerosis (UAAS) changes in chronic hemodialysis patients, but the underlying mechanisms remain unclear. Accordingly, we explore the protective role of $\mathrm{CSE} / \mathrm{H}_{2} \mathrm{~S}$ against uremic accelerated atherosclerosis and identify its possible signaling molecules in this study.

Methods: Six-week-old ApoE gene knockout $\left(\mathrm{ApoE}^{-/-}\right)$and wild-type (WT) mice were fed with either a control chow or atherogenic (high fat diet) diet for 10 weeks and subjected to chronic renal failure. The $\mathrm{H}_{2} \mathrm{~S}$ donor sodium hydrosulfide hydrate (NaHS) or an inhibitor of CSE D, L-propargylglycine (PPG) were administered to mice. Plasma lipid profile and atherosclerotic lesion size in the aortic roots were then analyzed. The protein expression level of CSE, TGF- $\beta$ and Smads were detected.
Results: ApoE ${ }^{-/-}$mice subjected to chronic renal failure developed early fatty lesions in the aortic root, elevated plasma levels of cholesterol and low-density lipoprotein cholesterol. Treatment of $\mathrm{ApoE}^{-/-}$mice with NaHS can inhibit the atherosclerosis development, but PPG administration can accelerate the atherosclerosis development. Meanwhile, the protein level of CSE, TGF- $\beta$ and Smads significantly increased in the $\mathrm{ApoE}^{-/}$mice subjected to chronic renal failure. Treatment of $\mathrm{ApoE}^{-/-}$mice with NaHS inhibits these protein decreases, but PPG treatment result in the increase of these proteins.

Conclusion: The $\mathrm{CSE} / \mathrm{H}_{2} \mathrm{~S}$ system is an important therapeutic target for protection against atherosclerosis, which may protect vascular tissues from atherogenic damage by regulating the TGF- $\beta / \mathrm{Smad}$ pathway.

Key Words: Uremic accelerated artherosclerosis, Hydrogen sulfide, TGF- $\beta /$ Smad pathway. 